\title{
THE UNCERTAINTY PRINCIPLE ON CAYLEY GRAPHS
}

\author{
ElinoR VELASQUEZ
}

\begin{abstract}
Heisenberg's uncertainty principle is extended to certain finite graphs. The fundamental theorem of calculus, integration by parts, and vanishing boundary terms for graphs are defined as well as functions of random variables, expectation values, and moments on graphs. Section 3 gives three versions of Heisenberg's uncertainty principle for graphs. For the 2nd version, we assume that our graph is the Cayley graph of a finite abelian group. We work out the example of a finite cycle graph in detail and compare it to the uncertainty principle on the continuous circle obtained by Grünbaum around 1990.
\end{abstract}

\section{Introduction.}

The classical Heisenberg uncertainty principle (see Weyl [16]) states that a particle's position, $x$, and momentum, $p_{x}$, cannot be simultaneously determined to an exact accuracy. We write this mathematically as

$$
\Delta x \Delta p_{x} \approx 2 \pi h
$$

where $h$ is a constant. In other words, a function and its Fourier transform cannot both be simultaneously concentrated on small intervals of the real line. Recently, work has been done to extend time- and band-limited versions of the principle to sets of small measure [6] and to sets contained in Gelfand pairs [1], [17], as well as to investigate nonlinear extensions of the classical principle [8]. In particular, the nonlinear situation, which involves a nonlinear Fourier transform, has applications in nuclear magnetic resonance related research.

Here we extend the uncertainty principle to a graph setting. Sometimes we will assume that our graph is a Cayley graph for a finite abelian group. Simply stated, a Cayley graph has a group action associated to its vertices and edges. The graph setting is used to describe the uncertainty principle in order to emphasize two themes. One theme or viewpoint is the connection between continuous and discrete mathematical models of physical situations. Note that finite Cayley graphs well illustrate finite difference schemes, objects associated to numerical solutions of differential equations. 
Often, continuous models of a physical situation have relied upon a group interpretation, typically by use of symmetry in the model's description, or in the solution of the associated differential equation. By working on Cayley graphs the group structure is retained in the discrete model. Another feature of the Cayley graph description in modeling physical situations: Cayley graphs naturally optimize grid and multigrid analysis of physical phenomena. This approach has been useful in studying the buckyball, a molecular model well-known to chemists: Lafferty and Rockmore [11] consider fast Fourier analysis on Cayley graphs associated to buckyballs. Hence the discretization of a physical problem is considerably improved. Another theme in the paper is that of statistical viewpoints on a Cayley graph, and again, considers the discretization of statistical models. We begin to develop some of these ideas with respect to finite data samples. Certainly, the use of group methods in statistics is not new. This paper attempts to mesh with the rapidly advancing area of statistics on algebraic structures.

In emphasizing the above themes, we hope to provide well-defined models useful for abelian and nonabelian discrete scattering theory and signal recovery. In other words, we hope to extend discrete scattering theory and discrete signal recovery from multigrid descriptions to the realm of grid spaces with non-zero curvatures, such as discrete spheres. The themes in this paper describe the methods relevant to these areas.

The original motivation was to further investigate the nonlinear version of the uncertainty principle described in Grünbaum [8]-[9]. The work presented in this paper represents the first half of this investigation: We have constructed the type of discretized model that is to be used in future work to describe the uncertainty inequality associated to solutions of differencedifferential equations and their associated scattering operators.

There are already many extensions of the uncertainty principle in the literature; we shall emphasize only the discrete extensions. Of recent interest has been the work of Donoho and Stark [6]. They obtain both a discrete and a continuous version of the time- and band-limited uncertainty inequality. Their finite uncertainty principle can be stated as:

$$
\text { If } f \in L^{2}\left(\mathbb{Z}_{n}\right) \text {, then }|\operatorname{supp} f| \cdot|\operatorname{supp} \hat{f}| \geq n,
$$

with supp $f$ denoting the support of $f,|A|$ denoting the cardinality of a set $A$, and $\hat{f}$ denoting the Fourier transform of $f$. Recently, the continuous version of the uncertainty principle from [6] has been extended by Wolf [17] to include the Gelfand pair setting. In Angel et al [1], we obtain finite analogues of the uncertainty principle for certain finite Gelfand pairs. Gelfand pairs associated with finite groups are automatically a type of finite Cayley graph. See Terras [15] for relevant commentary on this and as well as 
for applications regarding Donoho and Stark's theorems. An open problem is to construct the uncertainty inequality for cellular automata. We conclude by noting the existence of another discretized uncertainty principle: See Maassen [13] for a discretized entropic inequality.

\section{Cayley Graphs.}

Notation for graphs. We shall rely on Serre's notation [14] for graphs with a few variations. Describe an oriented graph $\Gamma$ by $\Gamma(V, E)$, with the set $V$ denoting the vertices of $\Gamma$ and set $E$ labeling the edges of $\Gamma$ such that the following set maps exist:

$$
E \rightarrow E, e \mapsto \bar{e} ; \quad E \rightarrow V \times V, \quad e \mapsto(\iota(e), \tau(e)),
$$

which satisfy $\bar{e} \neq e, \bar{e}=e, \iota(e)=\tau(\bar{e})$. The image $\bar{e}$ is the inverse of $e$. The image point $\iota(e)$ is called the origin of $e$, or incidence vertex, and $\tau(e)$ the terminal point of $e$, or end vertex. These maps, $\iota$ and $\tau$, are the incidence functions associated to $\Gamma$. We will definitely need to orient the edges of our graphs.

Consider the circle graph, $\mathbb{Z}_{n}$ as an illustration of the above notation. Fix $n \in \mathbb{N}$ and consider the graph $\Gamma$ such that the vertices are the elements from the group of integers modulo $n$, denoted by $\mathbb{Z}_{n}$, the edge set $E=$ $\{[i, i+1]\}_{i \in \mathbb{Z}_{n}}$, and the incidence functions $\iota([i, i+1])=i, \tau([i, i+1])=i+1$. Then, the orientation map in this case is $\theta: E \rightarrow \mathbb{Z}_{n} \times \mathbb{Z}_{n}, e \mapsto[\iota(e), \tau(e)]$.

Here the graph (and group) will always be finite. The set of functions on a subset $W$ of the set $V$ of vertices (or the set $E$ of edges) of our graph $\Gamma=\Gamma(V, E)$ will be denoted by $L^{2}(W)$. The notation for Cayley graphs is taken to be the following: Fix $G$ to be a finite group. Then, the Cayley graph $\Gamma=X(G, S)$ with respect to a fixed $S \subset G$ has $V=G$ and $E=G \times S$, with the incidence functions of $\Gamma: \iota(e)=\iota(g, s)=g, \tau(e)=\tau(g, s)=g s$ for all $e=(g, s) \in E$. Note that $\Gamma$ is connected if and only if $S$ is the generating set of $G$. See Bollobás [2], Cohen [3] or Dicks [5] for details. Also, define $\Gamma$ to be combinatorial whenever, given $S \subset G, S \cap S^{-1}=\emptyset$, meaning if $s \in S$, then $s^{-1} \notin S$. See Serre [14] for details. However, we shall not restrict $S$ to be necessarily the generating set for $G$ nor shall we require $\Gamma$ be combinatorial.

Heisenberg's uncertainty principle as formulated by Pauli and Weyl [16] relies on two ingredients: Integration by parts and the Schwarz inequality. To use an analogous method requires the construction of a one-form or derivative on a graph. Since the graphs are finite, a finite difference operator is used as the simplest discrete approximation to the continuous case. Other difference schemes can be chosen; consider the Lax-Friedrichs difference scheme as an example. In Lubotzky [12], a combinatorial Laplacian 
is considered for finite graphs. We use Lubotzky's construction of tangent vectors on a graph to define a difference operator.

Suppose $\Gamma=\Gamma(V, E)$ is a finite graph with a fixed orientation on its edges. Let $e=\left[e^{-}, e^{+}\right] \in E$ be an edge with $e^{-}$and $e^{+}$the incident vertex and end vertex, respectively. The edges of $\Gamma$ can be thought of as tangent vectors and $L^{2}(E)$ as 1-forms. The difference scheme $d: L^{2}(V) \rightarrow L^{2}(E)$ is defined by

$$
d f(e)=f\left(e^{+}\right)-f\left(e^{-}\right) .
$$

The incidence matrix $d$, with respect to canonical bases for $E \times V$, is

$$
d=[d]_{e, v}= \begin{cases}1, & v=e^{+} \\ -1, & v=e^{-} \\ 0, & \text { otherwise }\end{cases}
$$

We now present new analogs for the fundamental theorem of calculus and integration by parts on the graph $\Gamma$. Technically, the lemmas can be thought of as graph extensions of well known analogs for finite difference operators over the integers. Suppose $\Gamma$ to be connected for simplicity. Fix $P$ to be a path in the graph: $P=\Gamma\left(V^{\prime}, E^{\prime}\right) \subset \Gamma$. In other words, all edges and vertices are known, and because $\Gamma$ is finite, we can assign an ordering to $V^{\prime}$ and $E^{\prime}$. Suppose $P$ has $n$ edges: $E^{\prime}=\left\{e_{i}\right\}_{1}^{n}$ with $V^{\prime}=\left\{e_{i}^{-}, e_{i}^{+}\right\}_{1}^{n}$. We shall denote this path as $P=e_{1} \cup e_{2} \cup \cdots \cup e_{n}$. The cardinality of $V^{\prime}$ is $m \leq 2 n$.

Lemma 1 (The Fundamental Theorem of calculus on a finite graph). For $f \in L^{2}(E)$, and $P=e_{1} \cup e_{2} \cup \cdots \cup e_{n}$,

$$
\sum_{e \in P} d f(e)=f\left(e_{n}^{+}\right)-f\left(e_{1}^{-}\right) .
$$

Proof. Since $e_{i+1}^{-}=e_{i}^{+}$, for all $i$ with $1 \leq i \leq n-1$, the terms telescope:

$$
\begin{aligned}
\sum_{e \in P} d f(e) & =d f\left(e_{1}\right)+d f\left(e_{2}\right)+\cdots+d f\left(e_{n}\right) \\
& =f\left(e_{1}^{+}\right)-f\left(e_{1}^{-}\right)+f\left(e_{2}^{+}\right)-f\left(e_{2}^{-}\right)+\cdots+f\left(e_{n}^{+}\right)-f\left(e_{n}^{-}\right) \\
& =f\left(e_{n}^{+}\right)-f\left(e_{1}^{-}\right) .
\end{aligned}
$$

Lemma 2 (Integration by parts on a finite graph). Let $f, g \in$ $L^{2}(V)$ and $P=e_{1} \cup e_{2} \cup \cdots \cup e_{n}$. Then $\sum_{e \in P} d f(e) g\left(e^{+}\right)=[f(a) g(a)]_{e_{1}^{-}}^{e_{n}^{+}}-$ 
$\sum_{e \in P} f\left(e^{-}\right) d g(e)$.

Proof. Note that

$$
\sum_{e \in P}\left[f\left(e^{+}\right) g\left(e^{+}\right)-f\left(e^{-}\right) g\left(e^{-}\right)\right]=[f(a) g(a)]_{a=e_{1}^{-}}^{a=e_{n}^{+}},
$$

by the preceding Lemma. And,

$$
\begin{aligned}
& \sum_{e \in P}\left[f\left(e^{+}\right) g\left(e^{+}\right)-f\left(e^{-}\right) g\left(e^{-}\right)\right] \\
& =\sum_{e \in P}\left\{\left[f\left(e^{+}\right)-f\left(e^{-}\right)\right] g\left(e^{+}\right)+f\left(e^{-}\right)\left[g\left(e^{+}\right)-g\left(e^{-}\right)\right]\right\} \\
& =\sum_{e \in P}\left[d f(e) g\left(e^{+}\right)+f\left(e^{-}\right) d g(e)\right] .
\end{aligned}
$$

Vanishing boundary terms. Typically, Pauli and Weyl's proof [16] as well as Grünbaum's proof for the continuous circle [8] necessitate a vanishing of the boundary for the path $P$. In other words, throughout the rest of the paper we shall want the term

$$
[f(a) p(a)]_{e_{1}^{-}}^{e^{+}}
$$

to vanish. This can be easily accomplished by assuming that the support of $f \in L^{2}(G)$ does not include the endpoints.

Our result will also involve a parameterization function $p$ which will need to satisfy certain hypotheses. You can view $p$ as an analogue of the angle for the continuous circle.

Definition. The parameterization (or moment) function $p \in L^{2}(V)$ for a path $P$ on our graph must have values in $p(v) \in\{0,1,2, \ldots,|\Gamma|-1\}$ and satisfy the following hypotheses. For $\Gamma=\Gamma(E, V)$, let $P=e_{1} \cup e_{2} \cup \cdots \cup e_{n}$.

1) $d p(e) \geq 0$ for all but boundary $e \in E$; 2) $p\left(e_{1}^{-}\right)=0$.

Example. The finite circle. We can view the cycle graph or finite circle as the Cayley graph as $\Gamma\left(\mathbb{Z}_{n},\{1\}\right)$. Define

$$
p(k \bmod n)=k, \text { for } k=0,1, \ldots, n-1 .
$$

Orient the edges in the direction $e_{v}=[v, v+1(\bmod n)]$. Then $d p\left(e_{v}\right)=1$, except for $v=n-1$, where $d p\left(e_{n-1}\right)=-(n-1)$. If $f$ vanishes on the points 0 and $n-1$, this will not matter. 
If one seeks a parameterization function on a more general graph, one can, for example, choose a spanning tree, and use it to label the vertices outward from the base. Then this labeling will dictate the orientation of the edges.

Here we will concentrate on the case of finite circles, however.

Statistics on a graph. In discussing the Heisenberg uncertainty principle, Weyl [16] emphasizes the mean values or expectations of physical quantities for a quantum mechanical interpretation of a physical system as compared to a classical mechanical, non-probabilistic interpretation. With this viewpoint in mind, we frame our version of the uncertainty principle in terms of functions of random variables to emphasize the probabilistic content.

Let $(\Omega, U, P)$ be a measure space with total measure 1 . In other words, $(\Omega, U, P)$ is a probability space, $\Omega$ a nonempty set, $U$ a $\sigma$-algebra of subsets of $\Omega$, and $P$ a probability measure on $(\Omega, U)$. Let $\Gamma=\Gamma(E, V)$ be a finite graph of cardinality $n$. A measurable function $X: \Omega \rightarrow V$ is called a $\Gamma$-valued random variable with $P_{X}:=X(P)$ the distribution or probability measure. See Heyer [10] for details.

We proceed to set up the idea of random variables and expectation on a Cayley graph. First, we consider the finite group setting. Define a functional $E$ on $L^{2}(\Omega, \Gamma)$, e.g., the set of random variables, by

$$
E[X]=\sum_{\Omega} X d P
$$

with $P_{X}$ the distribution of $X$. For $\rho \in L^{2}(\Gamma)$ we have that

$$
E[\rho \circ X]=\sum_{g \in G} \rho(g) P_{\rho \circ X}(d g) .
$$

In general, we shall write

$$
E[\rho \circ X]=\sum_{g \in G} \rho(g) \mu_{X}(g),
$$

with $\mu_{X}$ as the distribution attached to the random variable $X$; in other words, $\mu_{X}(g)=P_{\rho \circ X}(d g)$, meaning $\mu_{X}(g)$ is the probability $X$ takes the value $g$. Sometimes we write this as $\mu_{X}(g)=P[X=g]$.

Moments on a finite graph. Fix $k \geq 0$. For $\rho \in L^{2}(\Gamma)$, suppose $R=\rho(X)$. Define the kth moments of the random function $R$ on $\Gamma$ by

$$
E\left[R^{k}\right]=E\left[(\rho \circ X)^{k}\right]=\sum_{g \in G}(\rho(g))^{k} \mu_{X}(g),
$$

with $\rho$ and $\mu_{X}$ as previously described. 
Moments on a Cayley graph. We next consider the situation on a Cayley graph. The projection map

$$
\pi: E \rightarrow G, \quad e \mapsto e^{+}
$$

for $e=\left[e^{-}, e^{+}\right] \in E$; we can use this map to extend both the random function and the probability distribution to the edge space $E=G \times S$ on the Cayley graph $\Gamma=\Gamma(G, G \times S)$. Fix $\alpha \in S$. Then, for $\rho \in L^{2}(G)$ and $\mu_{X}$ the distribution attached to $X \in L^{2}(\Omega)$, we extend to the edge space by

$$
\rho^{\alpha}:=\rho \circ \pi, \quad \mu_{X}^{\alpha}:=\mu_{X} \circ \pi .
$$

Therefore, extend the $k t h$ moments to the Cayley graph by defining for $R=\rho(X)$ :

$$
E\left[R^{k}\right]=\sum_{e \in E}\left(\rho^{\alpha}(e)\right)^{k} \mu_{X}^{\alpha}(e)=\sum_{e \in E}\left(\rho\left(e^{+}\right)\right)^{k} \mu_{X}\left(e^{+}\right)
$$

with regard to the probability $\mu_{X}$ attached to the random variable $X$. Suppose $k \geq 0$ is fixed.

\section{Uncertainty principles for graphs.}

Before we set up the uncertainty principle on a finite Cayley graph, we summarize the classical version of the uncertainty principle of Heisenberg as in Weyl [16]. Let $f \in L^{2}(\mathbb{R})$. We shall use the notation of moments. In Heisenberg's original inequality, the map $f$ is a wave function, and $|f(x)|^{2}$ is a probability distribution if $\|f\|_{2}^{2}=\int_{-\infty}^{+\infty}|f(x)|^{2} d x=1$. We will not assume this, however.

Suppose $X$ and $K$ are random variables. We have

$$
\left\langle X^{2}\right\rangle_{f}=\int_{-\infty}^{+\infty} x^{2}|f(x)|^{2} d x
$$

and similarly denote, with ${ }^{\wedge}$ the Fourier transform on $L^{2}(\mathbb{R})$,

$$
\left\langle K^{2}\right\rangle_{\hat{f}}=\int_{-\infty}^{+\infty} k^{2}|\hat{f}(k)|^{2} d k
$$

Requiring that the boundary term $[x f(x)]_{-\infty}^{+\infty}$ vanish, we then have the Heisenberg inequality for $\mathbb{R}^{1}$ :

$$
\frac{\left\langle X^{2}\right\rangle_{f}}{\|f\|_{2}^{2}} \frac{\left\langle K^{2}\right\rangle_{\hat{f}}}{\|\hat{f}\|_{2}^{2}} \geq \frac{1}{4},
$$


meaning, if $\left\langle K^{2}\right\rangle_{\hat{f}}$ is measured in a precise and accurate way, $\left\langle X^{2}\right\rangle_{f}$ cannot be precisely determined simultaneously.

The proof of this inequality comes from that of an equivalent inequality:

$$
\int_{-\infty}^{+\infty} x^{2}|f(x)|^{2} d x \int_{-\infty}^{+\infty} f^{\prime}(x) \overline{f^{\prime}(x)} d x \geq \frac{1}{4} \int_{-\infty}^{+\infty}|f(x)|^{2} d x
$$

Before discussing our uncertainty principle we need to consider some weighted $L^{2}$-norms for the functions on the vertices of our graph.

Weighted $L^{2}$ spaces. Let $p \in L^{2}(\Gamma)$ be the parameterization function satisfying (6). Suppose that $f \in L^{2}(\Gamma)$ is our wave function giving the state of a particle on the graph and assume it satisfies (5).

$$
\|f\|_{w}^{2}=\sum_{e \in E} d p(e)\left|f\left(e^{-}\right)\right|^{2} .
$$

Note that $\|f\|_{w}$ is a norm since we are assuming $d p(e) \geq 0$, whenever $f\left(e^{-}\right) \neq$ 0 . Of course all norms on the finite dimensional space $L^{2}(\Gamma)$ are equivalent. If our graph is a finite cycle graph, we assume that $d p(e)=1$ except on the edges where $f\left(e^{-}\right)=0$, and then we have $\|f\|_{w}^{2}=\|f\|^{2}=$ the usual $L^{2}$-norm.

The first version of our uncertainty principle is similar to (9). It makes no assumptions on the graph $\Gamma$ beyond the existence of $p, f \in L^{2}(\Gamma)$ satisfying (6), (5). That is, we do not need to assume that we have a group $G$. For the second version we will assume, in addition, that $\Gamma$ is a Cayley graph of an abelian group $G$.Of course, we are mainly interested here in finite circle graphs. As in Grünbaum [8], we assume that $f$ is real-valued is for simplicity.

Theorem 3 (Uncertainty principle \#1 for graphs). Suppose we have a finite graph $\Gamma=\Gamma(E, V)$. Assume that $p, f \in L^{2}(\Gamma)$ satisfy (6), (5), respectively. That is, we need to assume that

(i) $p(v) \in\{0,1, \ldots,|V|-1\} ; p\left(e_{1}^{-}\right)=0$;

(ii) $d p(e) \geq 0$, except where $f\left(e^{-}\right)=0$;

(iii) $f\left(e_{n}^{+}\right)=f\left(e_{n}^{-}\right)=0$ and $f$ is real-valued.

Then

$$
\sum_{e \in E}\left|f\left(e^{+}\right) p\left(e^{+}\right)\right|^{2} \sum_{e \in E}|d f(e)|^{2} \geq \frac{1}{4}\|f\|_{w}^{4},
$$

where

$$
\|f\|_{w}^{2}=\sum_{e \in E} d p(e) f\left(e^{-}\right)^{2} .
$$

Proof. First note that the Schwarz inequality implies that:

$$
\sum_{e \in E}\left|f\left(e^{+}\right) p\left(e^{+}\right)\right|^{2} \sum_{e \in E}|d f(e)|^{2} \geq\left(\sum_{e \in E}\left|p\left(e^{+}\right) f\left(e^{+}\right) d f(e)\right|\right)^{2} .
$$


Then

$$
\begin{aligned}
\sum_{e \in E} p\left(e^{+}\right) f\left(e^{+}\right) d f(e) & =\sum_{e \in E} p\left(e^{+}\right)\left[\left[f\left(e^{+}\right)\right]^{2}-f\left(e^{+}\right) f\left(e^{-}\right)\right] \\
& =\sum_{e \in E} p\left(e^{+}\right) \frac{1}{2}\left[\left[f\left(e^{+}\right)\right]^{2}-\left[f\left(e^{-}\right)\right]^{2}+[d f(e)]^{2}\right] \\
& =\frac{1}{2} \sum_{e \in E} p\left(e^{+}\right)\left[d\left[f^{2}\right](e)+[d f(e)]^{2}\right] \geq \frac{1}{2} \sum_{e \in E} p\left(e^{+}\right) d\left[f^{2}\right](e) \\
& \geq-\frac{1}{2} \sum_{e \in E} d p(e) f^{2}\left(e^{-}\right)=-\frac{1}{2}\|f\|_{w}^{2} .
\end{aligned}
$$

In the third to last step we used the assumption that $p\left(e^{+}\right) \geq 0$ (except perhaps where $d f(e)=0$ ). In the second to last step we used the Lemma on integration by parts proved earlier. It is here that we use the assumptions on $f$ and $p$ to see that the boundary terms vanish; i.e.,

$$
\left.f^{2}(a) p(a)\right|_{e_{1}^{-}} ^{e_{n}^{+}}=0
$$

The result follows. Note that on the finite circle $e_{n}^{+}=e_{1}^{-}$, which simplifies the hypotheses on $f$ a bit.

Next we seek a result more like (8). Thus we re-interpret our inequality for Cayley graphs of finite abelian groups $G$. Recall that the dual group $\widehat{G}$ consists of characters $\psi: G \rightarrow \mathbb{T}$ where $\mathbb{T}$ is the multiplicative group of complex numbers of norm 1 . The product on $\widehat{G}$ is pointwise. In fact the dual group is isomorphic to the original group when $G$ is finite abelian. The Fourier transform of $f \in L^{2}(G)$ is

$$
\widehat{f}(\psi)=\sum_{x \in G} f(x) \overline{\psi(x)} .
$$

It has all the properties of the usual Fourier transform; e.g., the shift formula obtained by setting $f^{s}(x)=f(x s)$, and noting that $\widehat{f^{s}}(\psi)=\psi(s) \widehat{f}(\psi)$. The Plancherel formula is

$$
\|f\|^{2}=\frac{1}{|G|}\|\hat{f}\|^{2}
$$

There is also an inversion formula. See Diaconis [4] and Terras [15] for the details.

Next we need to connect $\sum_{e \in E}|d f(e)|^{2}$ with the norm of the Fourier transform $\widehat{f}$. First, by definition:

$$
\sum_{e \in E}|d f(e)|^{2}=\sum_{s \in S} \sum_{x \in G}|f(x s)-f(x)|^{2} .
$$


Now use (12) to see that

$$
\sum_{e \in E}|d f(e)|^{2}=\frac{1}{|G|} \sum_{\psi \in \hat{G}} \sum_{s \in S}|\psi(s)-1|^{2}|\widehat{f}(\psi)|^{2} .
$$

We can define the second weighted norm on $F$ in $L^{2}(\hat{G})$ by

$$
\|F\|_{w^{\prime}}^{2}=\sum_{\psi \in \hat{G}} \sum_{s \in S}|\psi(s)-1|^{2}|F(\psi)|^{2}=\left\langle Q^{2}\right\rangle_{F} .
$$

Thus

$$
\sum_{e \in E}|d f(e)|^{2}=\frac{1}{|G|}\left\langle Q^{2}\right\rangle_{\widehat{f}}
$$

Theorem 4 (Uncertainty principle \#2 for finite Abelian Cayley graphs). With the same hypotheses as in the preceding Theorem plus the assumption that $G$ is abelian and $d p(e)=1$ except on the edges touching the boundary where $f$ vanishes, we have

$$
\frac{\left\langle P^{2}\right\rangle_{f}}{\|f\|^{2}} \frac{\left\langle Q^{2}\right\rangle_{\hat{f}}}{\|\hat{f}\|^{2}} \geq \frac{1}{4},
$$

where

$$
\left\langle P^{2}\right\rangle_{f}=\sum_{e \in E}\left|f\left(e^{+}\right) p\left(e^{+}\right)\right|^{2}
$$

and

$$
\left\langle Q^{2}\right\rangle_{\hat{f}}=\sum_{\psi \in \hat{G}} \sum_{s \in S}\left|\psi\left(s^{-1}\right)-1\right|^{2}|\widehat{f}(\psi)|^{2}
$$

Proof. The inequality follows from the preceding theorem and the intervening calculation.

Example. The Circle. We compare the discrete circle, $X=X\left(\mathbb{Z}_{n}, \mathbb{Z}_{n} \times\right.$ $\{1\})$, the circle graph, to the continuous circle $S^{1}=\left\{e^{i \theta} \mid-\pi \leq \theta \leq \pi\right\}$. Label the edges $e_{1}, \ldots, e_{n}$. Set $e_{i}^{-}=i-1(\bmod n)$, taking representatives of $\mathbb{Z}_{n}$ to be $0,1, \ldots, n-1$. Fix the parameterization: $p\left(e_{i}^{+}\right)=i$. Therefore, $d p\left(e_{i}\right)=p\left(e_{i}^{+}\right)-p\left(e_{i}^{-}\right)=i+1-i=1$, except for $i=n$. Assume that $f \in$ $L^{2}\left(\mathbb{Z}_{n}\right)$ is real-valued and vanishes at the endpoints in order to compare with the continuous situation. The representations on $\mathbb{Z}_{n}$ are the one-dimensional 
characters $\chi_{j}: \mathbb{Z}_{n} \rightarrow \mathbb{C}, \chi_{j}(k)=e^{\frac{2 \pi i j k}{n}}$, for $j=0, \ldots, n-1$. Take $S=\{1\}$ to define the edge set. Then we obtain

$$
\sum_{s \in S}\left|\chi_{j}(s)-1\right|^{2}=\left|\chi_{j}(1)-1\right|^{2}=\left|e^{\frac{2 \pi i j}{n}}-1\right|^{2}=4 \sin \left(\frac{\pi j}{n}\right)^{2} .
$$

Also,

$$
\left\langle Q^{2}\right\rangle_{\hat{f}}=4 \sum_{j=0}^{n-1}\left|\widehat{f}\left(\chi_{j}\right)\right|^{2} \sin \left(\frac{\pi j}{n}\right)^{2}
$$

Note also

$$
\|f\|_{w}^{2}=\frac{1}{n} \sum_{j=0}^{n-1}\left|c_{\chi_{j}}\right|^{2}
$$

by the Plancherel formula for groups. Therefore, the uncertainty principle for $f \in L^{2}\left(\mathbb{Z}_{n}\right)$, with $f\left(e_{n}^{ \pm}\right)=0$, looks like:

$$
\frac{\sum_{i=1}^{n-2}|f(i)|^{2} i^{2}}{\sum_{i=1}^{n-2}|f(i)|^{2}} \frac{4 \sum_{j=0}^{n-1}\left|\widehat{f}\left(\chi_{j}\right)\right|^{2} \sin \left(\frac{\pi j}{n}\right)^{2}}{\sum_{j=0}^{n-1}\left|\widehat{f}\left(\chi_{j}\right)\right|^{2}} \geq \frac{1}{4} .
$$

Compare the above to the case [8] of the continuous circle. The uncertainty principle for $f \in L^{2}\left(S^{1}\right)$ looks like:

$$
\frac{\int_{-\pi}^{\pi} f^{2}(\theta) \theta^{2} d \theta}{\int_{-\pi}^{\pi} f^{2}(\theta) d \theta} \frac{\sum_{-\infty}^{\infty} n^{2} c_{n}^{2}}{\sum_{-\infty}^{\infty} c_{n}^{2}} \geq \frac{1}{4}
$$

assuming that $f \in L^{2}\left(S^{1}\right)$ is real-valued, even and $f(\pi)=f(-\pi)=0$. Here $c_{n}$ denotes the nth Fourier coefficient of $f$.

Another uncertainty inequality. The following slightly different method to obtain an uncertainty inequality was proposed by the referee. If we proceed as in Grünbaum [8], who proved the uncertainty inequality for the continuous circle, we would start with real-valued $f$ and

$$
\sum_{e \in E} p\left(e^{+}\right) f\left(e^{+}\right) d f(e)=\left.f(a) f(a) p(a)\right|_{e_{1}^{-}} ^{e_{n}^{+}}-\sum_{e \in E} f\left(e^{-}\right) d(p \cdot f)(e) .
$$

Then use

$$
d(p \cdot f)=d p(e) f\left(e^{-}\right)+p\left(e^{+}\right) \cdot d f(e) .
$$

This gives, assuming the vanishing of $f$ at $e_{1}^{-}$and $e_{n}^{+}$:

$$
\sum_{e \in E} p\left(e^{+}\right)\left(f\left(e^{+}\right)+f\left(e^{-}\right)\right) d f(e)=-\sum_{e \in E} f\left(e^{-}\right)^{2} d p(e) .
$$


So define the weighted norm with the usual hypotheses on $f$ and $p$ :

$$
\|f\|_{w}^{2}=\sum_{e \in E} f\left(e^{-}\right)^{2} d p(e) .
$$

So far we have

$$
\sum_{e \in E} p(e) A v e(f) d f(e)=-\frac{1}{2}\|f\|_{w}^{2},
$$

upon setting the average value of $f$ on an edge equal to

$$
\text { Ave }(f)=\frac{1}{2}\left(f\left(e^{+}\right)+f\left(e^{-}\right)\right) .
$$

Then by the Schwarz inequality, we have the 3rd uncertainty inequality:

$$
\sum_{e \in E}\left(p\left(e^{+}\right) A v e(f)(e)\right)^{2} \sum_{e \in E} d f(e)^{2} \geq \frac{1}{4}\|f\|_{w}^{4} .
$$

Define another weighted norm of $f$ to be

$$
\|f\|_{p}^{2}=\sum_{e \in E}\left(p\left(e^{+}\right) \operatorname{Ave}(f)(e)\right)^{2} .
$$

Then we have proved, using the usual $L^{2}$-norm \|\| on $L^{2}(E)$, assuming that $f$ vanishes on $e_{1}^{-}$and $e_{n}^{+}$, and that $p$ satisfies $p(e) \geq 0$, except possibly on the first edge:

$$
\|f\|_{p}^{2}\|d f\|^{2} \geq \frac{1}{4}\|f\|_{w}^{4}
$$

Acknowledgments. The author would like to thank Audrey Terras and Joseph Wolf for the many discussions related to these results.

\section{References}

[1] J. Angel, S. Poulos, A. Terras, C. Trimble and E. Velasquez, Spherical functions and transforms on finite upper half planes: Eigenvalues of the combinatorial Laplacian, uncertainty, traces, Contemporary Math., 173, Amer. Math. Soc., Providence, (1994), 15-70.

[2] B. Bollobás, Graph theory: An introductory course, Springer-Verlag, N.Y., 1979.

[3] D.E. Cohen, Combinatorial group theory: A topological approach, Cambridge U. P., New York, 1989.

[4] P. Diaconis, Group representations in probability and statistics, Math. Inst. Stat., Hayward, CA, 1986.

[5] W. Dicks and M.J. Dunwoody, Groups acting on graphs, Cambridge U. P., New York, 1989. 
[6] D.L. Donoho and P.B. Stark, Uncertainty principles and signal recovery, SIAM J. Appl. Math., 49 (1989), 906-931.

[7] J.L. Gross and T.W. Tucker, Topological graph theory, Wiley-Interscience, New York, 1987.

[8] F.A. Grünbaum, Trying to beat Heisenberg, Lecture Notes in Pure and Appl. Math., 122 (1990), Dekker, New York, 657-665.

[9]_, Concentrating a potential and its scattering transform for a discrete version of the Schrödinger and Zakharov-Shabat operators, Physica D, 44 (1990), 92-98.

[10] H. Heyer, Probability measures on locally compact groups, Springer-Verlag, New York, 1977.

[11] J. Lafferty and D. Rockmore, Fast Fourier analysis for $S L_{2}$ over a finite field and related numerical experiments, Experimental Math., 1 (1992), 115-139.

[12] A. Lubotzky, Discrete groups, expanding graphs and invariant measures, Birkhäuser, Basel, 1994.

[13] H. Maassen, A discrete entropic uncertainty relation, in Quantum probability and applications, V, Springer-Verlag, New York, (1988), 263-266

[14] J.-P. Serre, Trees, Springer-Verlag, New York, 1980.

[15] A.A. Terras, Fourier analysis on finite groups and applications, in preparation.

[16] H. Weyl, The theory of groups and quantum mechanics, Dover, New York, 1928.

[17] J.A. Wolf, Uncertainty principles for Gelfand pairs and Cayley complexes, in '75 Years of Radon transform' (Ed. S. Gindikin and P. Michor), International Press, Boston, (1994), 271-292.

Received September 2, 1993 and revised February 2, 1997.

UNIVERSITY OF CALIFORNIA

BERKELEY, CA 94720

E-mail address: velasquez@math.berkeley.edu 